\title{
Scarless Wound Closure by a Mussel-Inspired Poly(amidoamine) Tissue Adhesive with Tunable Degradability
}

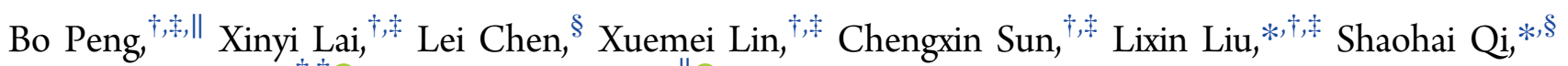
Yongming Chen, $*, \dagger,+\left({ }^{\circ}\right.$ and Kam W. Leong ${ }^{\| \bullet}$

${ }^{\dagger}$ Center of Functional Biomaterials, School of Material Science and Engineering, and ${ }^{\star}$ Key Laboratory for Polymeric Composite and Functional Materials of Ministry of Education, Sun Yat-sen University, Guangzhou 510275, Guangdong, China

${ }^{\S}$ Department of Burns Surgery, The First Affiliated Hospital of Sun Yat-sen University, Guangzhou 510080, Guangdong, China

"Department of Biomedical Engineering, Columbia University, New York, New York 10025, United States

\section{Supporting Information}

ABSTRACT: Burn, trauma, and various medical conditions including bacterial infection, diabetes complication, and surgery could lead to an acute cutaneous wound and scar formation. Application of tissue glues instead of sutures could minimize the additional trauma and scar formation. Despite the countless efforts devoted to the development of high-strength tissue glues, little attention has been paid to their influence on the scar formation. Here, we report the development of a new tissue glue with excellent biocompatibility and tunable degradability for scarless wound closure. A series of catecholcontaining poly(amidoamine) (CPAA) polymers were synthesized via the one-step Michael addition of dopamine and bisacrylamide. The tertiary amino group in the polymer backbone was used to introduce a zwitterionic sulfobetaine group by one-step ringopening polymerization. The introduction of the zwitterionic sulfobetaine group could easily tune the hydrophilicity and the degradability of CPAA without influencing the density of the catechol group in the polymer. Lap-shear tests on the porcine skin demonstrated a high adhesion strength of $7 \mathrm{kPa}$ at $1 \mathrm{~h}$, rising to $24 \mathrm{kPa}$ by $12 \mathrm{~h}$. Addition of silica nanoparticles could further enhance the adhesion strength by $50 \%$. In vivo studies further confirmed that the CPAA tissue glue could effectively accelerate the healing process of incisional wounds on the back of Sprague Dawley rats compared with suture and reduce the scar formation.

\section{INTRODUCTION}

Every year, millions of patients need a proper wound closure procedure to enable healing, and a large proportion of them eventually acquire scars. ${ }^{1}$ Scar is a dysregulated response to cutaneous wound healing, including excessive proliferation of fibroblasts and abnormal deposition of collagen. Scar formation goes beyond aesthetics, disfigurement, or movement restriction, sometimes even leading to cancer. ${ }^{2,3}$ Numerous research efforts have been made for scarless wound healing; application of tissue glues is one promising strategy. ${ }^{4,5}$ Compared with the traditional wound closure techniques of using sutures and staples, tissue glues provide faster wound closure, no additional trauma, less inflammation, and reduced scar formation. ${ }^{6-13}$ The reduced scar length and morbidity make this technology particularly appealing in cosmetic surgeries, such as facelift surgery. ${ }^{14,15}$ Cyanoacrylate is the first kind of commercialized tissue glues that could rapidly polymerize on contact with water or blood. However, the cyanoacrylate glue fails to gain widespread popularity because of its variable outcome and tissue toxicity. ${ }^{16}$ Besides cyanoacrylate, tissue glues based on fibrin are also commercially available. Although they have the advantages of fast curing and biodegradability, the use of fibrin glues of human origin encompasses risks of blood-borne disease transmission and potential allergic reactions. ${ }^{17}$

In recent years, it is found that some marine organisms including barnacles and marine mussels show the ability of affixing themselves to virtually all types of surfaces under aqueous conditions. The strong adhesive property of mussel proteins comes from the unusual amino acid 3,4-dihydroxyphenylalanine (DOPA). Through oxidative cross-linking or metal chelation, the catechol moiety in DOPA could form a strong adhesive layer between various surfaces. ${ }^{18-20}$ To mimic this natural adhesive, many kinds of polymers carrying catechol groups have been developed, including polypeptides, ${ }^{21,22}$ polyacrylates, ${ }^{23-25}$ poly(ethylene glycol)s (PEGs), ${ }^{26-28}$ poly(ester urea)s, ${ }^{29}$ polyurethane, ${ }^{30}$ and polystyrene-based systems. ${ }^{31-34}$ Becker et al. ${ }^{29}$ have synthesized poly(ester urea)s by interfacial polymerization, and the catechol groups were introduced via 1-ethyl-3-(3-dimethylaminopropyl)carbodiimide condensation between the phenol group and the acetonideprotected 3,4-dihydroxyhydrocinnamic acid. These polymers

Received: August 21, 2017

Accepted: September 8, 2017

Published: September 21, 2017 
show a strong adhesive strength which is comparable to the commercialized fibrin glue on the porcine skin. Messersmith et al. $^{35-38}$ have developed multiple tissue adhesive systems. One of the most successful systems is the four-arm PEG end-capped with catechol groups. It achieves high adhesive strength, low toxicity in vitro, and excellent wound closure ability in vivo.

However, the influence of the catechol-containing tissue glue on the scar formation has been studied rarely. The aim of this research is to develop a biodegradable tissue glue that could achieve safe and scarless wound closure. Poly(amidoamine) is a class of biodegradable polymers that have been widely used for biomedical applications because of their structure similar to that of polypeptide, good biodegradability, and biocompatibility. ${ }^{39,40}$ Herein, catechol-containing poly(amidoamine) (CPAA) and catechol-containing poly(amino ester) (CPAE) were synthesized through the one-step Michael addition reaction of commercialized monomers. Because poly(amidoamine) has an inherent tertiary amino group, each repeating unit could serve as a bifunctional group. Zwitterionic groups could be introduced into the polymer through the one-step ring-opening reaction of 1,3-propane sultone without influencing the density of catechol groups in the polymer. The introduction of highly hydrophilic zwitterionic sulfobetaine groups could systematically tune the hydrophilicity and degradation rate of CPAA. CPAA could be used as a tissue glue after the catechol group was cross-linked by the $\mathrm{Fe}^{3+}$ ion. Lap-shear tests proved that CPAA had adequate adhesion strength. When the polymer was applied to the rat skin wound closure, the wound healing rate was accelerated and the scar formation was reduced compared with both the suture and the commercialized fibrin glue (see Schemes 1 and 2).

\section{RESULTS AND DISCUSSION}

Polymer Synthesis and Characterization. Synthesis of the catechol-containing polymer is a challenging task. The catechol-containing monomers are usually made from

\section{Scheme 1. Application of $\mathrm{Fe}^{3+}$-Cross-Linked CPAA as a Tissue Glue for Scarless Wound Closure}

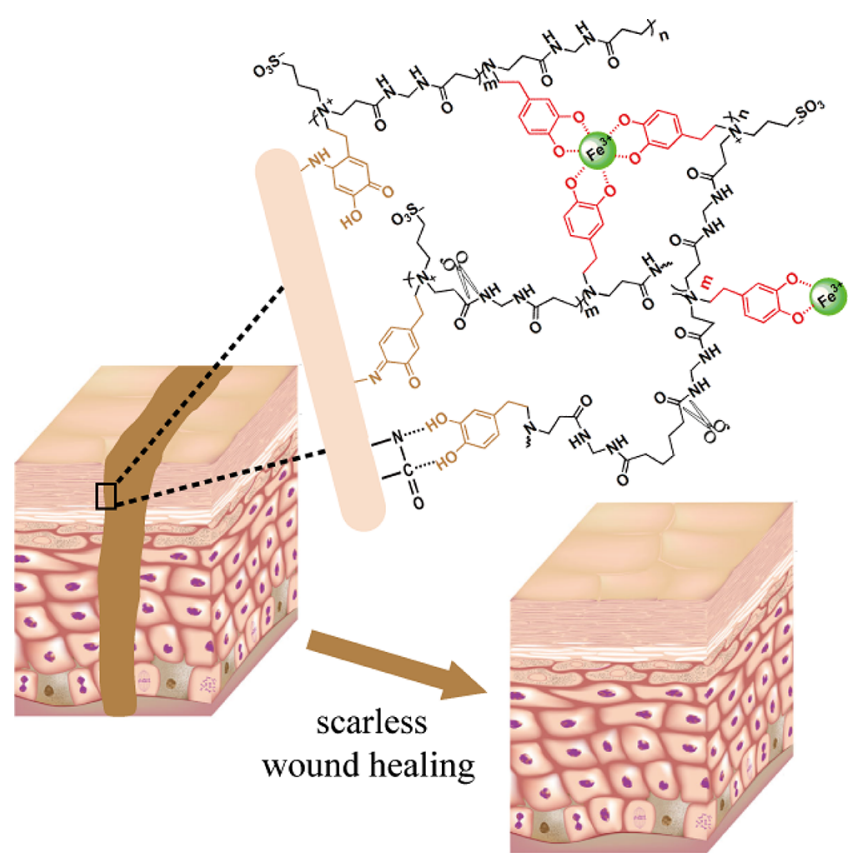

dopamine that is oxygen-sensitive. Complicated protection and deprotection steps are required to prevent the oxidation of dopamine during the reaction and purification processes. ${ }^{15}$ Although postmodification can be adopted to prepare catecholcontaining polymers, the low reactivity of the amino group in dopamine makes this approach inefficient. ${ }^{16}$ The ease of poly(amidoamine) synthesis by polycondensation should, in principle, facilitate the structure-property elucidation of bioadhesive polymers. Herein, the one-step Michael addition reaction was chosen to synthesize CPAA and CPAE.

CPAA and CPAE were synthesized under similar conditions to compare the influence of the monomer on the adhesive strength and degradability. PEG diacrylates with different EG repeating units were chosen to prepare CPAE to study the influence of the length of diacrylates on their adhesive strength. The aminoamide and amino ester bond in CPAA and CPAE will also lead to a different degradability of the tissue glue. The CPAE-1,2 and 3 were using monomer with 2, 8 and 11 EO units. Size exclusion chromatography (SEC) analysis showed that CPAA and CPAE were synthesized with a molecular weight ranging from 20000 to 30000 (Table 1 and Figure S1). ${ }^{1} \mathrm{H}$ NMR spectra of the purified CPAA and CPAE are depicted in Figure 1. The successful incorporation of catechol groups into CPAA and CPAE was confirmed by the characteristic peaks of the catechol group at 6.45 and $6.65 \mathrm{ppm}$, respectively. In CPAA, the peaks at around 2.2, 2.5, and $4.3 \mathrm{ppm}$ were attributed to the methylene protons of $N, N^{\prime}$-methylene bis(acrylamide) (Figure 1A). In CPAE, the peaks at around $3.5,3.6$, and $4.1 \mathrm{ppm}$ were attributed to the methylene protons of PEG in the PEG diacrylate (Figure $1 \mathrm{~B}-\mathrm{D}$ ).

The hydrophilicity of polymers determines the number of water molecules bounded to the polymers. An increase of hydrophilicity would facilitate the access of water molecules to the polymer backbone, resulting in accelerated hydrolysis of the polymer. The introduction of a hydrophilic group into the polymer could potentially tune its degradation rate. The zwitterionic sulfobetaine group with a strong water-binding ability ${ }^{41}$ was chosen to augment the hydrophilicity of polymers in this study. The tertiary amine groups could be used to introduce the zwitterionic sulfobetaine groups by the one-step ring-opening reaction. A series of CPAA-ZS with different hydrophilicities was synthesized through the ring-opening reaction between CPAA and 1,3-propane sultone (Table 2). In addition, the reaction conversion could be controlled through adjusting the reaction time and the molar ratio of 1,3-propane sultone to tertiary amine. The conversion was determined by the characteristic peak of the methylene group in the zwitterionic sulfobetaine at $2.1 \mathrm{ppm}$ (Figure S2).

Adhesion Strength of Polymers with Different Crosslinking Densities and Hydrophilicities. Catechol groups can form stable linkages by introducing the cross-linker, $\mathrm{FeCl}_{3}$, to the system. However, the optimal molar ratio of catechol groups to $\mathrm{FeCl}_{3}$ needs to be investigated. Herein, the lap-shear adhesion strength of CPAA mixed with different amounts of $\mathrm{FeCl}_{3}$ was measured to investigate the optimal molar ratio of catechol groups to $\mathrm{FeCl}_{3}$ on the porcine skin. Dimethyl sulfoxide (DMSO) was chosen to increase the solubility of CPAA because it is widely used in biomedical applications. ${ }^{42,43}$ As shown in Figure 2A, when the molar ratio of CPAA to $\mathrm{FeCl}_{3}$ varied from 10:1 to 10:4, the adhesion strength first increased from $2.8 \mathrm{kPa}$ (catechol $/ \mathrm{FeCl}_{3}=10: 1$ ) to $3.9 \mathrm{kPa}$ (catechol/ $\mathrm{FeCl}_{3}=10: 2$ ) and then decreased to around $2 \mathrm{kPa}$ (catechol/ $\mathrm{FeCl}_{3}=10: 3$ and 10:4). This can be explained by the 
Scheme 2. Synthesis Route of CPAA and CPAA Modified with Zwitterionic Sulfobetaine (CPAA-ZS)

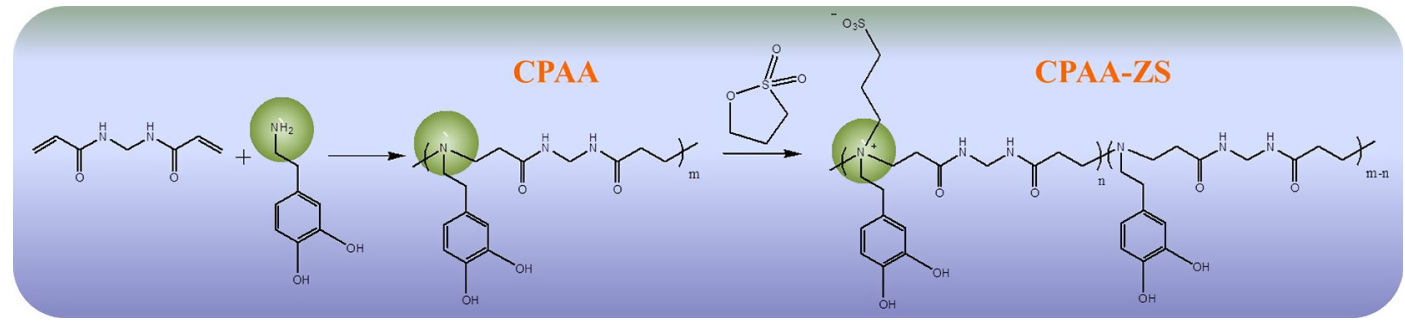

Table 1. Characterization of CPAA and CPAE

$\begin{array}{lcc}\text { polymer }^{a} & M_{\mathrm{n}}{ }^{b} & M_{\mathrm{w}} / M_{\mathrm{n}}{ }^{b} \\ \text { CPAA } & 23900 & 1.12 \\ \text { CPAE-1 } & 22200 & 1.16 \\ \text { CPAE-2 } & 21400 & 1.21 \\ \text { CPAE-3 } & 29600 & 1.27\end{array}$

${ }^{a_{T}}$ The polymerizations were all conducted in DMSO at $50{ }^{\circ} \mathrm{C}$ for $5 \mathrm{~d}$. ${ }^{b}$ Measured by SEC using polystyrene standards for calibration.

competition between catechol and $\mathrm{Fe}^{3+}$ : multiple bidentate complexation and single bidentate complexation. ${ }^{44}$

Then, the wet adhesion strength properties of CPAA and CPAE synthesized by PEG diacrylate with different EG repeating units were investigated. As shown in Figure $2 \mathrm{~B}$, the wet adhesion strength of CPAE was all around $1 \mathrm{kPa}$, which was much lower than that of CPAA. This is because the density of the catechol group in the CPAE backbone is lower than that in CPAA, which could be attributed to the longer chain length of the PEG diacrylate than $N, N^{\prime}$-methylene bis(acrylamide). The influence of hydrophilicity, finely tuned by the
Table 2. Preparation of CPAA-ZS

\begin{tabular}{cccc} 
polymer $^{a}$ & {$[\mathrm{CPAA}] /[1,3$-propane sultone $]$} & time $(\mathrm{h})$ & $\begin{array}{c}\text { conversion } \\
(\%)\end{array}$ \\
\hline CPAA-ZS-11 & $1: 1$ & 10 & 11 \\
CPAA-ZS-15 & $1: 1$ & 20 & 15 \\
CPAA-ZS-18 & $1: 1$ & 30 & 18 \\
CPAA-ZS-21 & $1: 1$ & 40 & 21 \\
CPAA-ZS-34 & $1: 3$ & 30 & 34 \\
CPAA-ZS-53 & $1: 3$ & 40 & 53
\end{tabular}

${ }^{a_{\text {The }}}$ ring-opening reactions were all conducted in DMF at $45{ }^{\circ} \mathrm{C}$.

introduction of zwitterionic sulfobetaine groups, was also investigated (Figure 2C). Although the hydrophilicity of the polymer was greatly enhanced after the ring-opening reaction, the wet adhesion strength was not compromised by the introduction of zwitterionic sulfobetaine groups in the polymers. When the grafting ratio reached $21 \%$, the tissue glue showed the highest adhesion strength $(3.4 \mathrm{kPa})$.

Because the polymer could be adsorbed and adhered to the surface of inorganic nanoparticles, the addition of inorganic nanoparticles was widely used to increase the gel cohesion
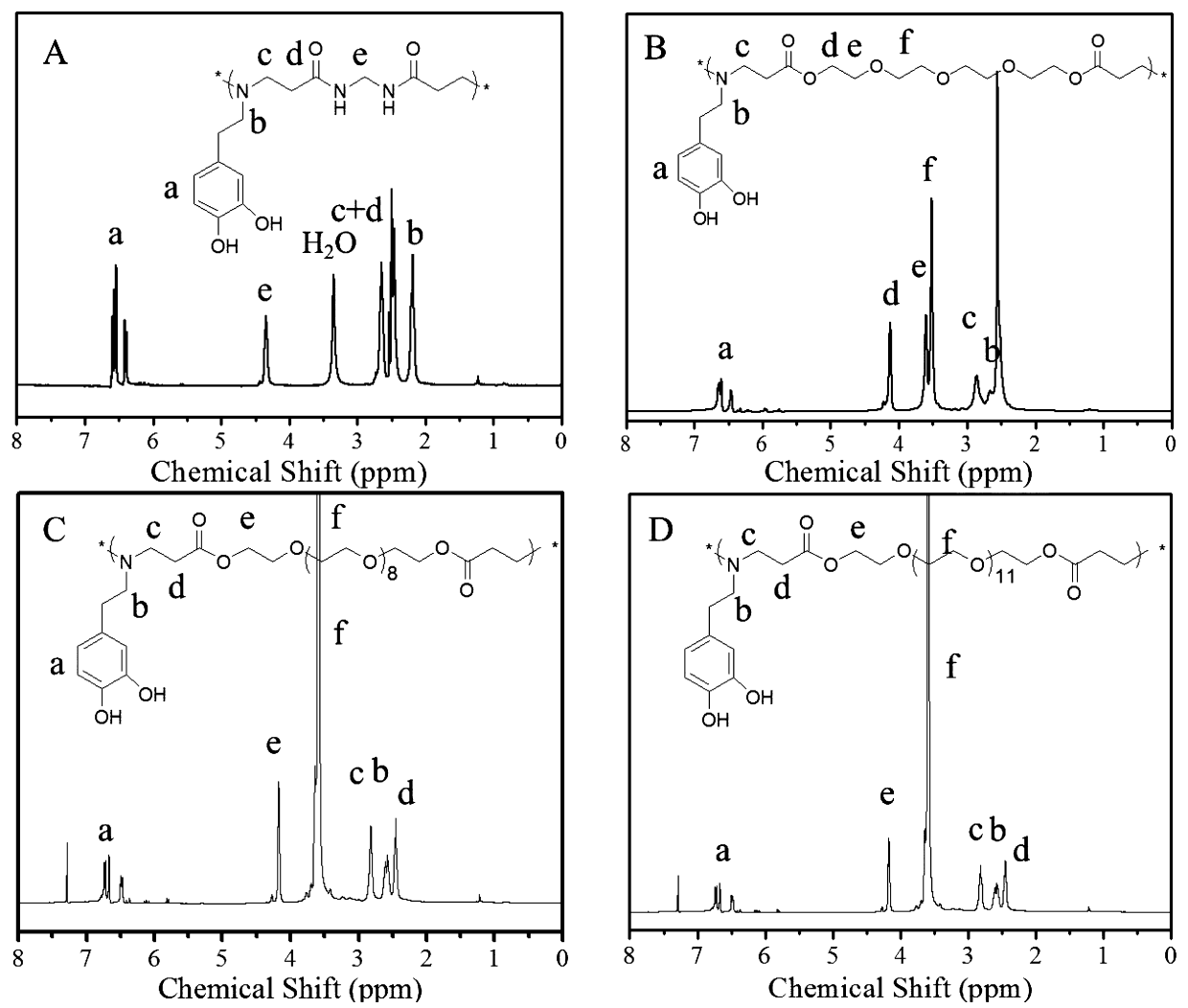

Figure 1. ${ }^{1} \mathrm{H}$ NMR spectra of (A) CPAA and (B) CPAE-1 in $d$-DMSO; (C) CPAE-2 and (D) CPAE-3 in $\mathrm{CDCl}_{3}$. 

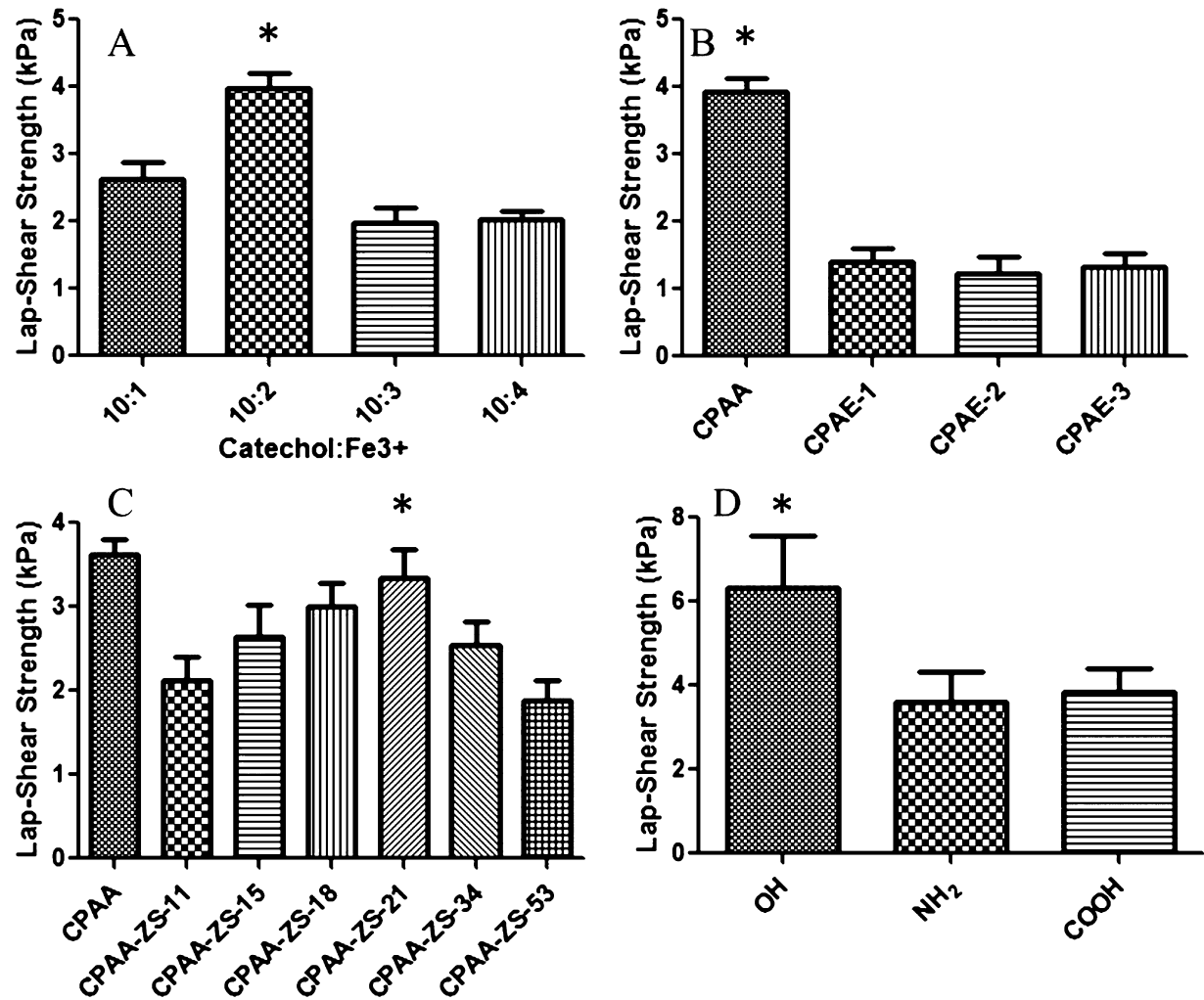

Figure 2. Lap-shear strength of (A) CPAA cross-linked by different amounts of $\mathrm{FeCl}_{3}$; (B) CPAA, CPAE-1, CPAE-2, and CPAE-3 at a catechol/ $\mathrm{FeCl}_{3}$ ratio of 5; (C) CPAA-ZS with different ring-opening efficiencies; and (D) CPAA tissue glue with a catechol/FeCl 3 ratio of 5 blended with $2 \%$ of $500 \mathrm{~nm}$ silica nanoparticles with different functional groups on the surface. The error bars represent SD $(n=5)$, and the statistics are determined by $t$-test $(* P<0.05)$.
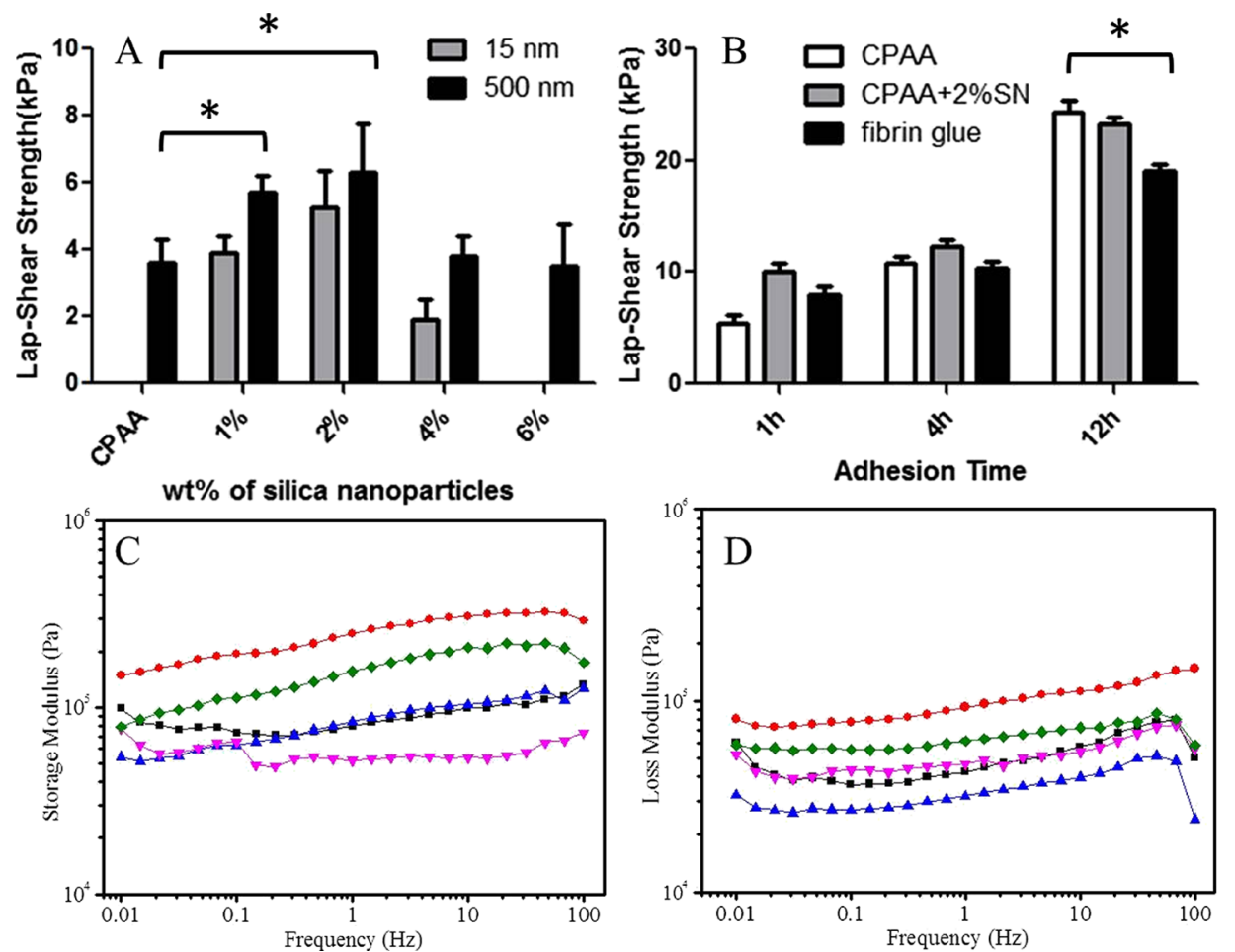

Figure 3. Lap-shear strength of (A) CPAA and CPAA blend with silica nanoparticles with the diameters of $15 \mathrm{~nm}$ (gray) and $500 \mathrm{~nm}$ (black) at different concentrations; (B) CPAA (white), CPAA blend with $2 \%$ of $500 \mathrm{~nm}$ silica nanoparticles (gray), and fibrin glue (black) at different adhesion times. The error bars represent SD $(n=3)$, and the statistics are determined by $t$-test $\left({ }^{*} P<0.05\right)$. (C) storage and (D) loss moduli of the CPAA tissue glue containing $1 \mathrm{wt} \%$ (black) and $2 \mathrm{wt} \% 15 \mathrm{~nm}$ (red) silica nanoparticles and $1 \mathrm{wt} \%$ (pink) and $2 \mathrm{wt} \% 500 \mathrm{~nm}$ (green) silica nanoparticles and the silica-free CPAA tissue glue (blue) subjected to an oscillatory strain of 0.1 at a frequency of $0.1-100 \mathrm{~Hz}$. 
strength. $^{45}$ For instance, the addition of Laponite could significantly increase the adhesion strength and the toughness of the tissue glue. ${ }^{28}$ Because of the excellent biocompatibility and controllable diameter of the silica nanoparticles, they were chosen as the reinforcing additive. ${ }^{46,47}$ The influence of the functional groups on the surface of silica nanoparticles was first investigated. The bare silica nanoparticles could greatly increase the adhesion strength of the polymers, whereas silica nanoparticles functionalized with an amino group or a carboxyl group could not exhibit the same property (Figure 2D). It might be because the surface modification of silica nanoparticles hindered the access of DOPA to the silica nanoparticles.

The concentration and the diameter of silica nanoparticles also had a significant impact on the reinforcement performance. When the concentration of silica nanoparticles was increased from 1 to $6 \%$, the wet adhesion strength first increased and then began to decrease after culminating at $2 \%$. When the concentration was increased from 0 to $2 \%$, the internal crosslink point increased, so the strength of the tissue glue increased. However, when the silica nanoparticle concentration was further increased from 2 to $6 \%$, they could not disperse well in the tissue glue, and the aggregation of silica nanoparticles decreased the strength of the tissue adhesive. For $15 \mathrm{~nm}$ silica nanoparticles, the highest concentration they would achieve is $4 \%$. Further increase of concentration will result in a serious coagulum, so we did not test the adhesive strength. By comparing the lap-shear adhesion strengths of the polymers blended with silica nanoparticles with different diameters, it could be seen from Figure $3 \mathrm{~A}$ that $500 \mathrm{~nm}$ silica nanoparticles performed better than the $15 \mathrm{~nm}$ nanoparticles.

As shown in Figure 3B, the adhesion strength of the CPAA tissue glue was comparable with that of the fibrin glue in the first $4 \mathrm{~h}$. Then, the adhesion strength of the CPAA tissue glue increased and exhibited a better performance than the fibrin glue. The adhesive strength of the DOPA-based tissue adhesive comes from the thiol group in the tissue which could react with the tissue glue. The longer adhesion time could help more covalent bond formation between the tissue and the tissue glue, so the adhesive strength increased with time.

To further understand the influence of silica nanoparticles in the bioadhesives, the viscoelastic property of the tissue glue was determined using oscillatory rheometry (Figure 3C,D). For all formulations tested, the storage modulus $\left(G^{\prime}\right)$ was greater than the loss modulus $\left(G^{\prime \prime}\right)$, indicating that the hydrogel was chemically cross-linked. The CPAA tissue glue without silica nanoparticles showed $70 \mathrm{kPa}$ storage modulus and $30 \mathrm{kPa}$ loss modulus at around $1 \mathrm{~Hz}$. Both the storage and the loss moduli increased with the frequency. The addition of 1 wt $\%$ silica nanoparticles significantly increased the loss modulus of the CPAA tissue glue but not the storage modulus. The increased loss modulus indicated an enhanced viscous dissipation property, which might be attributed to the presence of reversible hydrogen bonds between the CPAA polymer and silica nanoparticles in the hydrogel network. The unchanged storage modulus indicated that the addition of $1 \mathrm{wt} \%$ silica nanoparticles did not change the structure of the hydrogel network. By contrast, 2 wt \% of silica nanoparticles significantly enhanced both the storage and the loss moduli. Unlike the adhesion strength, the $15 \mathrm{~nm}$ silica nanoparticles displayed higher storage and loss moduli. This can be explained by the competition between the silica-catechol interaction and the skin-catechol interaction. The smaller silica nanoparticles with a larger surface area would adsorb more catechol groups, which would decrease the number of active catechol groups for adhesion.

Degradation and Cytotoxicity of the Polymers. Because of the influence of nitrogen in the backbone, the amide bonds in the backbone of poly(amidoamine)s are very vulnerable to degradation in aqueous solution. ${ }^{48}$ Because the regeneration abilities of different organs are quite different, the application of tissue glues in different organs requires different degradation rates. A series of tissue glues with a tunable degradation rate could be an ideal candidate to fulfill the requirement under a variety of situations. Herein, the degradability of CPAA and CPAA-ZS was evaluated in vitro at $\mathrm{pH}=7.4$ and $37{ }^{\circ} \mathrm{C}$ for 22 days; the mass loss profiles are shown in Figure 4. After 22 days, CPAA degraded less than

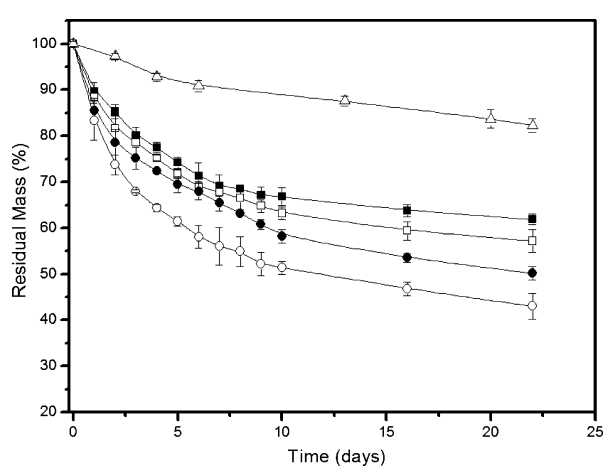

Figure 4. Degradation of CPAA (- $\triangle$-), CPAA-ZS-11 (- - $)$, CPAAZS-15 (- $\square-)$, CPAA-ZS-18 (-๑), and CPAA-ZS-21 (-O-) at pH = 7.4 and $37^{\circ} \mathrm{C}$.

$20 \%$ of its original mass. The introduction of the zwitterionic sulfobetaine accelerated the degradation, bringing the mass loss to 38 and $57 \%$ with $11 \%$ (CPAA-ZS-11) and $21 \%$ (CPAA-ZS21 ) of sulfobetaine incorporated into the polymer, respectively, in the same period. Because of the vulnerability of the amino ester bond in CPAE, all CPAE lost more than $70 \%$ weight within 3 days, which makes them not suitable for in vivo applications.

Macrophages are one of the key players in the wound healing process. They are responsible for clearing debris, releasing cytokines, and stimulating fibroblast division, collagen synthesis, and angiogenesis. ${ }^{19}$ The cytotoxicity of CPAA and CPAA-ZS was evaluated in the RAW 264.7 mouse macrophage cell line (Figure 5). The cells attached and proliferated on the substrate coated with CPAA (Figure 5B) or CPAA-ZS (Figure S3) ${ }^{49}$ The cytotoxicity of the dissolution content was evaluated by the Transwell system. The $\mathrm{Fe}^{3+}$-cross-linked tissue glue (30 $\mu \mathrm{L}$ ) made from CPAA or CPAA-ZS was put into the Transwell chamber and cocultured with the RAW 264.7 cells for $24 \mathrm{~h}$. No cytotoxicity was detected, which proves that the introduction of zwitterionic sulfobetaine did not affect the cytotoxicity.

In Vivo Cutaneous Incisional Wound Healing Study. The cutaneous incisional wound healing model ${ }^{50}$ was used to evaluate the in vivo performance of the CPAA tissue glue. The wound healing process could be divided into four overlapping but well-defined phases: hemostasis, inflammation, proliferation, and remodeling and scar formation. The perfect tissue glue should be able to achieve immediate wound closure and hemostasis, reduce the wound healing time, and minimize the scar formation. During the animal study, the CPAA tissue glue 

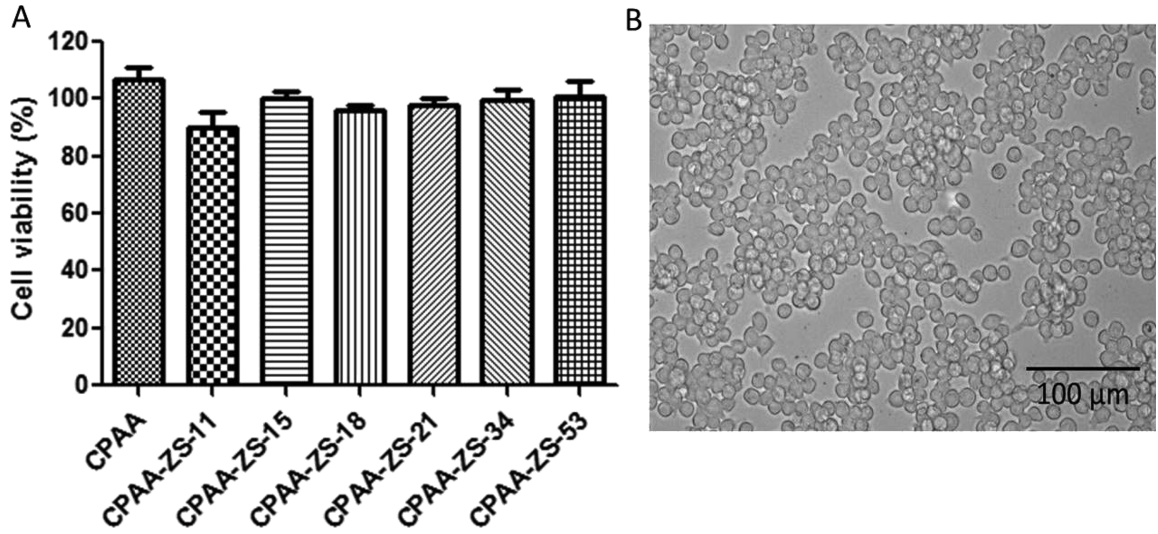

Figure 5. (A) Cytotoxicity of the $\mathrm{Fe}^{3+}$-cross-linked CPAA and CPAA-ZS tissue glues on the RAW 264.7 cells. The error bars represent SD $(n=3)$. (B) Microscopy image of the RAW 264.7 cells grown on a CPAA-coated 24-well plate.

or fibrin glue was applied to the wound openings on the dorsum of Sprague Dawley rats after cleaning the blood. In the control group, the wounds were closed by sutures. The morphological examination of the CPAA glue, fibrin glue, and suture groups is shown in Figure 6. Application of the fibrin

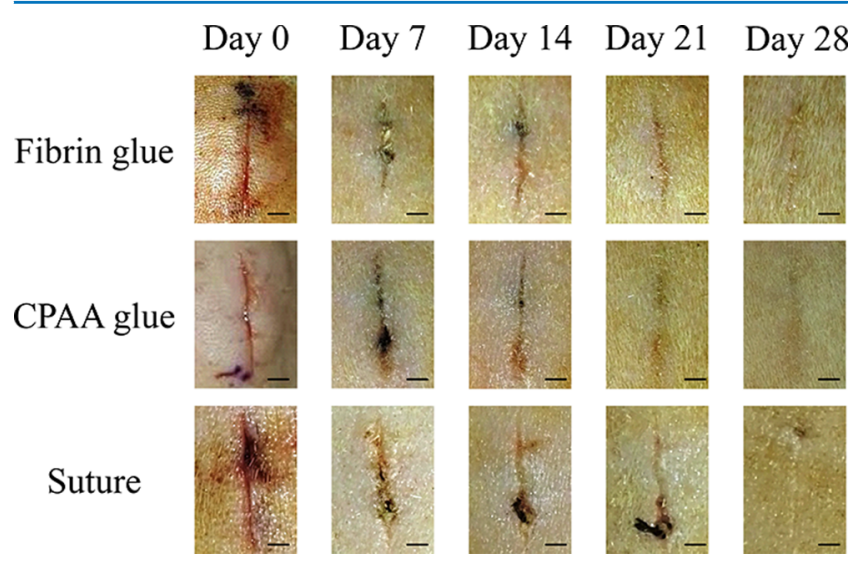

Figure 6. Morphological comparison of fibrin glue, CPAA glue, and suture in the Sprague Dawley rat model; the scale bar is $0.3 \mathrm{~cm}$.

glue and the CPAA glue could achieve both wound closure and hemostasis. On day 14, the fibrin glue and the CPAA glue exhibited a similar wound recovery, outperforming the suture group. On day 21, the wounds in the CPAA glue group almost fully recovered, whereas the wound scar could be clearly seen in the fibrin glue group and the suture group. On day 28 , all groups showed full recovery of the wound, and the CPAA glue group showed a lower degree of scar formation compared with the fibrin glue group.

Detailed wound healing process analysis was performed on day 28 through hematoxylin and eosin (H\&E) staining. For normal wound healing, day 28 should be the end of the proliferative phase and in the middle of remodeling and scar formation process. $H \& E$ staining (Figure 7A) showed severe immune cell infiltration in the suture-treated group, whereas the CPAA glue and fibrin glue both showed little immune cell infiltration (Figure 7B,C). The area of underlying scar tissue of the CPAA glue-treated group $\left(0.13 \pm 0.00 \mathrm{~mm}^{2}\right)$ is much smaller than those of the other two groups at day 28 (suturetreated group: $0.83 \pm 0.01 \mathrm{~mm}^{2}$ and fibrin glue-treated group: $0.24 \pm 0.02 \mathrm{~mm}^{2}$; Figure $7 \mathrm{G}$ ). Masson's trichrome staining and analysis was applied to depict collagen deposition and organization in the incisional wound area. Whereas the collagen fibers in the suture-treated group and the fibrin glue-treated group were still in an admixed packed state (Figure 7D,E), more organized collagen (brighter blue) fibers were present in the CPAA glue-treated group at day 28 (Figure $7 F$ ). During the remodeling phase, the collagen reorganization and degradation occur simultaneously to provide tensile strength and reduce scar formation. Quantitative image analysis (Figure $7 \mathrm{H}$ ) demonstrated that the intensity of collagen deposition in the CPAA glue-treated group $(0.30 \pm 0.02)$ was significantly lower than those in the suture-treated group $(0.44 \pm 0.02)$ and the fibrin glue-treated group $(0.42 \pm 0.02)(P<0.05)$. These in vivo results indicated that the application of the CPAA glue led to a scarless wound closure in the cutaneous incisional wound model.

\section{CONCLUSIONS}

A new type of mussel-inspired biodegradable CPAA polymer adhesives was synthesized using the one-step Michael addition reaction. CPAA exhibited low cytotoxicity, controlled degradability, and reasonable wet tissue adhesion strength in vitro. The comparison between CPAA and CPAE indicated that the high adhesion strength of CPAA may be due to the higher catechol density in the CPAA backbone. Blending with silica nanoparticles could further enhance its adhesion strength. The introduction of zwitterionic sulfobetaine groups by the ringopening reaction could tune the hydrophilicity and degradation rate of polymers without jeopardizing their adhesion performance. In vivo studies further proved that the CPAA glue could achieve efficient wound closure, which was characterized by accelerated wound healing and decreased scar formation. With the advantageous adhesion strength, tunable degradability, and promising performance in the in vivo wound healing rat model, the CPAA-based biomimetic adhesive might provide a new clinical option for wound closure in which scar formation is the primary consideration such as in cosmetic surgery.

\section{EXPERIMENTAL SECTION}

Materials. Dopamine hydrochloride (99\%, Sigma-Aldrich), PEG diacrylate $\left(M_{\mathrm{n}}=302,575\right.$, and $700 \mathrm{Da}, 99 \%$, Alfa Aesar), $N, N^{\prime}$-methylene bis(acrylamide) (99\%, Alfa Aesar), 1,3propane sultone (99\%, Alfa Aesar), sodium bromide (99\%, Alfa Aesar), and other chemicals were used as received. Silica nanoparticles were purchased from Aladdin. A commercialized fibrin tissue adhesive was purchased from Shanghai Raas Blood 

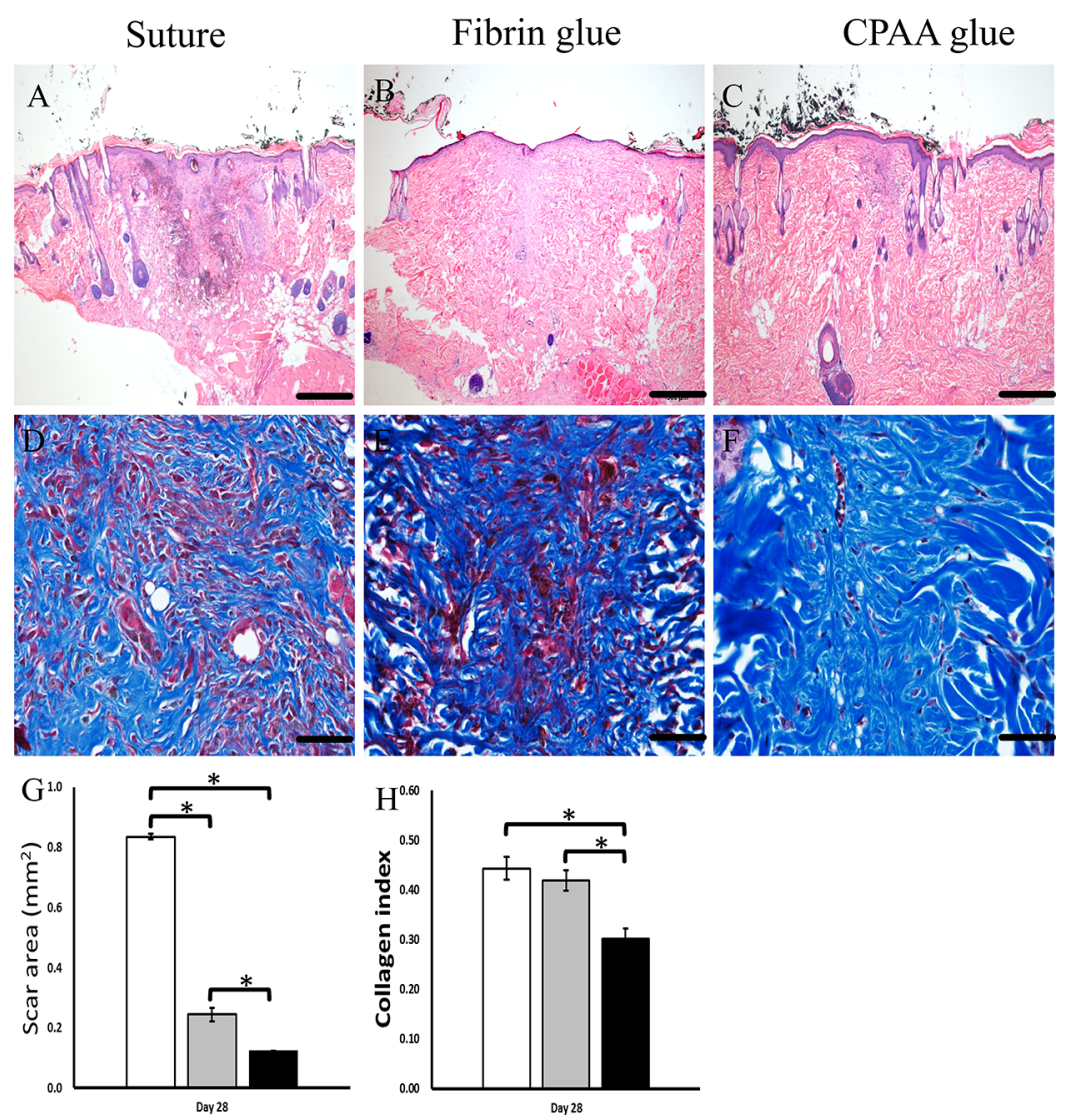

Figure 7. H\&E staining and Masson's trichrome staining of wounds at day 28. H\&E staining of (A) suture, (B) fibrin glue-treated, and (C) CPAA glue-treated wounds. Masson's trichrome staining of (D) suture, (E) fibrin glue-treated, and (F) CPAA glue-treated wounds. Quantitative analysis of scar areas through $(\mathrm{G}) \mathrm{H} \& \mathrm{E}$ staining and $(\mathrm{H})$ Masson's trichrome staining. The scale bar is $500 \mu \mathrm{m}$. The error bars represent SD ( $n=3)$, and the statistics are determined by $t$-test $(* P<0.05)$.

Products Co. Ltd. (Shanghai, China). In cell culture, Dulbecco's modified Eagle's medium (DMEM) was obtained from Corning. Fetal bovine serum (FBS), penicillin/streptomycin (pen/strep), and phosphate-buffered saline (PBS, $\mathrm{pH}$ 7.4) were obtained from Gibco BRL.

Synthesis of CPAA and CPAE Polymers via the Michael Addition Reaction. As a general procedure, $N, N^{\prime}$ methylene bis(acrylamide) (1.54 g, $10 \mathrm{mmol})$, triethylamine (4 $\mathrm{mL})$, and DMSO $(10 \mathrm{~mL})$ were discharged into a $50 \mathrm{~mL}$ Schlenk flask. The mixture was degassed by two freezeevacuate-thaw cycles, and then dopamine hydrochloride (1.96 g, $10 \mathrm{mmol}$ ) was discharged into the Schlenk flask. The mixture was further degassed by three evacuate-thaw cycles, and the Schlenk flask was sealed. Polymerization was carried out in an oil bath thermostated at $50{ }^{\circ} \mathrm{C}$ for $5 \mathrm{~d}$. After polymerization, the solution was distilled under vacuum to remove the excess DMSO. For CPAA, the resulting product was purified by dialysis in water for $3 \mathrm{~d}$, and then a light yellow solid was obtained after lyophilization (yield: $88 \%$ ). For CPAE, the resulting product was purified by precipitating it into diethyl ether three times.

Synthesis of CPAA-ZS via the Ring-Opening Reaction of 1,3-Propane Sultone. In a $25 \mathrm{~mL}$ round-bottom flask equipped with a magnetic stir bar, CPAA $(0.7 \mathrm{~g})$, 1,3-propane sultone $(0.8 \mathrm{~g})$, and sodium bromide $(0.08 \mathrm{~g})$ were stirred to dissolve in $10 \mathrm{~mL}$ of dimethyl formamide (DMF). The solution was stirred at $45{ }^{\circ} \mathrm{C}$ for $45 \mathrm{~h}$. Then, the solution was concentrated by a rotary evaporator before precipitating it two times in tetrahydrofuran. The resulting product was purified by dialysis in water for $2 \mathrm{~d}$, and a white powder was obtained after lyophilization. The composition was determined by the ${ }^{1} \mathrm{H}$ NMR spectrum.

Characterization. ${ }^{1} \mathrm{H}$ NMR spectra were recorded on a Bruker AVANCE III 400-NMR spectrometer at room temperature. SEC was performed by a set of an Agilent 1260 pump, an Agilent 1260 refractometer, and three PLgel columns (mixed$\mathrm{D}$, mixed-D, and mixed-B) using DMF as an eluent at a flow rate of $1.0 \mathrm{~mL} \cdot \mathrm{min}^{-1}$ at $35^{\circ} \mathrm{C}$. Polystyrene standards were used for calibration.

Adhesion Strength Measurement. Lap-shear tensile stress measurements were performed on the porcine skin following the procedures described in ASTM standard F225505 . Fresh porcine skin was obtained from the slaughterhouse and then stocked at $-20{ }^{\circ} \mathrm{C}$ before use. A small piece of the thawed porcine skin was cut into rectangular pieces with dimensions of $5.0 \mathrm{~cm} \times 1.5 \mathrm{~cm}$ after removing excess fat, and then these pieces were immersed in PBS $(\mathrm{pH}=7.4)$ for immediate use. Tissue glues were prepared by adding $20 \mu \mathrm{L}$ of $\mathrm{FeCl}_{3}$ solution into $20 \mu \mathrm{L}$ of $30 \mathrm{wt} \%$ polymer solutions in DMSO with different concentrations at $\mathrm{pH}=8.5$. The mixtures were treated with ultrasound for $20 \mathrm{~min}$. Then, the tissue glues were smeared between two partially overlapped porcine skins with an overlapping area of $2 \mathrm{~cm} \times 1 \mathrm{~cm}$. The adhesive joints were compressed with $2.5 \mathrm{kPa}$ pressure for $5 \mathrm{~min}$. Then, the adhesive joints were pulled at a rate of $5 \mathrm{~mm} \cdot \mathrm{min}^{-1}$ until the two porcine skins were separated, using a floor-standing electromechanical universal testing machine (SUNS 
UTM5000 Series, Shenzhen, China). Each measurement was repeated five times and averaged.

Dynamic Rheological Tests. Dynamic rheological tests of the CPAA tissue glue were carried out at room temperature using a Rheometric Scientific HAAKE (Thermo Fisher Scientific) strain-controlled rheometer equipped with $35 \mathrm{~mm}$ parallel plates. The storage modulus $\left(G^{\prime}\right)$ and the loss tangent (the ratio of loss modulus $G^{\prime \prime}$ to storage modulus $G^{\prime}$ ) of the tissue glue were determined at the frequency ranging from 0.1 to $100 \mathrm{~Hz}$ at 0.1 strain amplitude. All rheological measurements were performed in triplicate.

Degradation Study. Degradation was assessed by the percentage of weight loss over a period of 1 month. The CPAA polymer was prepared in a $7 \mathrm{~mL}$ glass vial (each empty glass vial was weighed) and immersed in PBS $(\mathrm{pH}=7.4)$ in an incubator at $37^{\circ} \mathrm{C}$. At regular intervals, after removing PBS and washing the CPAA polymer with distilled water, the vial with CPAA was dried and weighed again. The overall mass loss was calculated by deducting the recorded empty vial weight from the final mass values.

The CPAA-ZS polymers were dissolved in $3 \mathrm{~mL}$ of deionized water and transferred into a dialysis bag (molecular weight cutoff $=1000)$. Then, the polymer was dialyzed at $37^{\circ} \mathrm{C}$ for 22 d. Then, the solution was freeze-dried to get the remaining polymer, and the weight was recorded. Each polymer was tested three times.

Cell Culture and Cytotoxicity Study in Vitro. The cytotoxicity of the dissolution content of the tissue glue was measured using a quantitative MTT assay. RAW 264.6 mouse macrophages were cultured in DMEM containing 10\% FBS and 10 units $\cdot \mathrm{mL}^{-1}$ pen/strep at $37{ }^{\circ} \mathrm{C}$ in a $5 \% \mathrm{CO}_{2}$ humidified atmosphere. Cells were seeded in a 24 well-plate at a concentration of 40000 cells per well for $24 \mathrm{~h}$. The tissue glue $(30 \mu \mathrm{L})$ was added into the Transwell chamber. The cells cultured in DMEM were set as a control. After incubation for $24 \mathrm{~h}$, the Transwell chamber and the medium were removed and replaced with $50 \mu \mathrm{L}$ of MTT solution $\left(1 \mathrm{mg} \cdot \mathrm{mL}^{-1}\right.$ in PBS), and the cells were incubated for another $4 \mathrm{~h}$. Finally, the MTT solution was removed, and $100 \mu \mathrm{L}$ of DMSO was added per well to dissolve the crystals completely. The absorbance of each well at $570 \mathrm{~nm}$ was measured using a Multiskan FC microplate reader (Thermo Fisher, USA). For the microscopy image of RAW 264.7, first, a solution of CPAA or CPAA-ZS in the Tris buffer $\left(\mathrm{pH}=8.5,2 \mathrm{mg} \cdot \mathrm{mL}^{-1}\right)$ was added into the 24 well-plate and coated for $24 \mathrm{~h}$. After the solution was removed, the polymers were coated on the bottom of the plate. Then, the cells were seeded and cultured for $24 \mathrm{~h}$ before taking the image.

Animal Study for Incisional Wound Closure. All in vivo experiments and animal care were approved by the Institutional Animal Care and Use Committee of the Sun Yat-sen University ([2014] C-031). To evaluate the tissue glue property and the biocompatibility of the CPAA tissue adhesive, rats (normal Sprague Dawley rat, 180-220 g, 3 months, male) were randomly divided into three groups, five rats in each group. The rats were anesthetized using an isoflurane-oxygen gas mixture, and fur around the surgery site was removed. Skin incisions $(1.5 \mathrm{~cm}$ long and full skin thickness deep) were made on their back. The skin incisions were quickly closed by the interrupted suture, fibrin glue, and CPAA glue. The CPAA tissue glue (50 $\mu \mathrm{L}$ ) was applied to the wound area, and the wound was pressed by tweezers for $1 \mathrm{~min}$. At 28 days after wound closure, the incisions and the surrounding normal skin were harvested and fixed in $p$-formaldehyde solution (3.7 wt \%) for histological analysis by H\&E staining and Masson's trichrome staining. Measurements of scar areas and collagen index in high-power field were performed by ImageJ software. ${ }^{51,52}$

\section{ASSOCIATED CONTENT}

\section{Supporting Information}

The Supporting Information is available free of charge on the ACS Publications website at DOI: 10.1021/acsomega.7b01221.

SEC traces of CPAA and CPAE, ${ }^{1} \mathrm{H}$ NMR spectrum of CPAA-ZS-53 in DMSO- $d_{6}$, and microscopy images of RAW 264.7 cells (PDF)

\section{AUTHOR INFORMATION}

\section{Corresponding Authors}

*E-mail: liulixin@mail.sysu.edu.cn (L.L.).

*E-mail: qishaohaigzburns@163.com (S.Q.).

*E-mail: chenym35@mail.sysu.edu.cn (Y.C.).

ORCID 웅

Yongming Chen: 0000-0003-2843-5543

Kam W. Leong: 0000-0002-3269-5770

\section{Author Contributions}

The manuscript was written through the contributions of all authors. All authors have given approval to the final version of the manuscript.

\section{Notes}

The authors declare no competing financial interest.

\section{ACKNOWLEDGMENTS}

We acknowledge funding support by National Natural Science Foundation of China (grant no. 51403243), the China Postdoctoral Science Foundation (grant no. 2014M552262), International Postdoctorate Exchange Program, Guangdong Innovative and Entrepreneurial Research Team Program (grant no. 2013S086), and the Natural Science Foundation of Guangdong province, China (grant no. 2014A030312018). We also acknowledge the support of the Guangdong Technical Engineering Research Center of Functional Biomaterials.

\section{REFERENCES}

(1) Frost, S. J.; Mawad, D.; Hook, J.; Lauto, A. Micro- and Nanostructured Biomaterials for Sutureless Tissue Repair. Adv. Healthc. Mater. 2016, 5, 401-414.

(2) Bobba, R. K.; Holly, J. S.; Loy, T.; Perry, M. C. Scar Carcinoma of the Lung: A Historical Perspective. Clin. Lung Cancer 2011, 12, 148154.

(3) Horton, C. E.; Crawford, H. H.; Love, H. G.; Loeffler, R. A. The Malignant Potential of Burn Scar. Plast. Reconstr. Surg. 1958, 22, 348353.

(4) Biddlestone, J.; McGill, D. Untied horizontal mattress and wound glue to reduce scarring in simple paediatric wounds. J. Plast. Reconstr. Aesthet. Surg. 2017, 70, 133-134.

(5) Norouzi, M.; Boroujeni, S. M.; Omidvarkordshouli, N.; Soleimani, M. Advances in Skin Regeneration: Application of Electrospun Scaffolds. Adv. Healthc. Mater. 2015, 4, 1114-1133.

(6) Ahn, B. K.; Das, S.; Linstadt, R.; Kaufman, Y.; MartinezRodriguez, N. R.; Mirshafian, R.; Kesselman, E.; Talmon, Y.; Lipshutz, B. H.; Israelachvili, J. N.; Waite, J. H. High-performance musselinspired adhesives of reduced complexity. Nat. Commun. 2015, 6, 8663.

(7) Hong, S.; Pirovich, D.; Kilcoyne, A.; Huang, C.-H.; Lee, H.; Weissleder, R. Supramolecular Metallo-Bioadhesive for Minimally Invasive Use. Adv. Mater. 2016, 28, 8675-8680. 
(8) Lih, E.; Lee, J. S.; Park, K. M.; Park, K. D. Rapidly curable chitosan-PEG hydrogels as tissue adhesives for hemostasis and wound healing. Acta Biomater. 2012, 8, 3261-3269.

(9) Kim, B. J.; Oh, D. X.; Kim, S.; Seo, J. H.; Hwang, D. S.; Masic, A.; Han, D. K.; Cha, H. J. Mussel-Mimetic Protein-Based Adhesive Hydrogel. Biomacromolecules 2014, 15, 1579-1585.

(10) Bouten, P. J. M.; Zonjee, M.; Bender, J.; Yauw, S. T. K.; van Goor, H.; van Hest, J. C. M.; Hoogenboom, R. The chemistry of tissue adhesive materials. Progr. Polym. Sci. 2014, 39, 1375-1405.

(11) Giano, M. C.; Ibrahim, Z.; Medina, S. H.; Sarhane, K. A.; Christensen, J. M.; Yamada, Y.; Brandacher, G.; Schneider, J. P. Injectable bioadhesive hydrogels with innate antibacterial properties. Nat. Commun. 2014, 5, 4095.

(12) Shi, Y.; Zhou, P.; Jérôme, V.; Freitag, R.; Agarwal, S. Enzymatically Degradable Polyester-Based Adhesives. ACS Biomater. Sci. Eng. 2015, 1, 971-977.

(13) Silvestri, A.; Brandi, C.; Grimaldi, L.; Nisi, G.; Brafa, A.; Calabrò, M.; D'Aniello, C. Octyl-2-Cyanoacrylate Adhesive for Skin Closure and Prevention of Infection in Plastic Surgery. Aesthet. Plast. Surg. 2006, 30, 695-699.

(14) Berry, M. G.; Stanek, J. J. Fibrin tissue adhesive for face- and necklift. J. Plast. Reconstr. Aesthet. Surg. 2015, 68, 1325-1331.

(15) Toriumi, D. M.; O’Grady, K.; Desai, D.; Bagal, A. Use of Octyl2-Cyanoacrylate for Skin Closure in Facial Plastic Surgery. Plast. Reconstr. Surg. 1998, 102, 2209-2219.

(16) Chow, A.; Marshall, H.; Zacharakis, E.; Paraskeva, P.; Purkayastha, S. Use of Tissue Glue for Surgical Incision Closure: A Systematic Review and Meta-Analysis of Randomized Controlled Trials. J. Am. Coll. Surgeons 2010, 211, 114-125.

(17) Traver, M. A.; Assimos, D. G. New generation tissue sealants and hemostatic agents: innovative urologic applications. Rev. Urol. 2006, 8, 104-111.

(18) Lee, B. P.; Messersmith, P. B.; Israelachvili, J. N.; Waite, J. H. Mussel-Inspired Adhesives and Coatings. Annu. Rev. Mater. Res. 2011 , 41, 99-132.

(19) Yang, J.; Stuart, M. A. C.; Kamperman, M. Jack of all trades: versatile catechol crosslinking mechanisms. Chem. Soc. Rev. 2014, 43, 8271-8298.

(20) Lee, H.; Dellatore, S. M.; Miller, W. M.; Messersmith, P. B. Mussel-Inspired Surface Chemistry for Multifunctional Coatings. Science 2007, 318, 426-430.

(21) Wang, J.; Liu, C.; Lu, X.; Yin, M. Co-polypeptides of 3,4dihydroxyphenylalanine and l-lysine to mimic marine adhesive protein. Biomaterials 2007, 28, 3456-3468.

(22) Wei, W.; Petrone, L.; Tan, Y.; Cai, H.; Israelachvili, J. N.; Miserez, A.; Waite, J. H. An Underwater Surface-Drying Peptide Inspired by a Mussel Adhesive Protein. Adv. Funct. Mater. 2016, 26, 3496-3507.

(23) Lee, H.; Lee, B. P.; Messersmith, P. B. A reversible wet/dry adhesive inspired by mussels and geckos. Nature 2007, 448, 338-341.

(24) Nishida, J.; Kobayashi, M.; Takahara, A. Light-Triggered Adhesion of Water-Soluble Polymers with a Caged Catechol Group. ACS Macro Lett. 2013, 2, 112-115.

(25) Nishida, J.; Kobayashi, M.; Takahara, A. Gelation and adhesion behavior of mussel adhesive protein mimetic polymer. J. Polym. Sci. Part A: Polym. Chem. 2013, 51, 1058-1065.

(26) Cencer, M.; Murley, M.; Liu, Y.; Lee, B. P. Effect of NitroFunctionalization on the Cross-Linking and Bioadhesion of Biomimetic Adhesive Moiety. Biomacromolecules 2015, 16, 404-410.

(27) Lee, B. P.; Dalsin, J. L.; Messersmith, P. B. Synthesis and Gelation of DOPA-Modified Poly(ethylene glycol) Hydrogels. Biomacromolecules 2002, 3, 1038-1047.

(28) Liu, Y.; Meng, H.; Konst, S.; Sarmiento, R.; Rajachar, R.; Lee, B. P. Injectable Dopamine-Modified Poly(ethylene glycol) Nanocomposite Hydrogel with Enhanced Adhesive Property and Bioactivity. ACS Appl. Mater. Interfaces 2014, 6, 16982-16992.

(29) Zhou, J.; Defante, A. P.; Lin, F.; Xu, Y.; Yu, J.; Gao, Y.; Childers, E.; Dhinojwala, A.; Becker, M. L. Adhesion Properties of Catechol-
Based Biodegradable Amino Acid-Based Poly(ester urea) Copolymers Inspired from Mussel Proteins. Biomacromolecules 2015, 16, 266-274.

(30) Sun, P.; Wang, J.; Yao, X.; Peng, Y.; Tu, X.; Du, P.; Zheng, Z.; Wang, X. Facile Preparation of Mussel-Inspired Polyurethane Hydrogel and Its Rapid Curing Behavior. ACS Appl. Mater. Interfaces 2014, 6, 12495-12504.

(31) Meredith, H. J.; Jenkins, C. L.; Wilker, J. J. Enhancing the Adhesion of a Biomimetic Polymer Yields Performance Rivaling Commercial Glues. Adv. Funct. Mater. 2014, 24, 3259-3267.

(32) Matos-Pérez, C. R.; Wilker, J. J. Ambivalent Adhesives: Combining Biomimetic Cross-Linking with Antiadhesive Oligo(ethylene glycol). Macromolecules 2012, 45, 6634-6639.

(33) Matos-Pérez, C. R.; White, J. D.; Wilker, J. J. Polymer Composition and Substrate Influences on the Adhesive Bonding of a Biomimetic, Cross-Linking Polymer. J. Am. Chem. Soc. 2012, 134, 9498-9505.

(34) Jenkins, C. L.; Meredith, H. J.; Wilker, J. J. Molecular Weight Effects upon the Adhesive Bonding of a Mussel Mimetic Polymer. ACS Appl. Mater. Interfaces 2013, 5, 5091-5096.

(35) Fullenkamp, D. E.; Rivera, J. G.; Gong, Y.-k.; Lau, K. H. A.; He, L.; Varshney, R.; Messersmith, P. B. Mussel-inspired silver-releasing antibacterial hydrogels. Biomaterials 2012, 33, 3783-3791.

(36) Brubaker, C. E.; Kissler, H.; Wang, L.-J.; Kaufman, D. B.; Messersmith, P. B. Biological performance of mussel-inspired adhesive in extrahepatic islet transplantation. Biomaterials 2010, 31, 420-427.

(37) Brubaker, C. E.; Messersmith, P. B. Enzymatically Degradable Mussel-Inspired Adhesive Hydrogel. Biomacromolecules 2011, 12, 4326-4334.

(38) Barrett, D. G.; Bushnell, G. G.; Messersmith, P. B. Mechanically Robust, Negative-Swelling, Mussel-Inspired Tissue Adhesives. Adv. Healthc. Mater. 2013, 2, 745-755.

(39) Ferruti, P. Poly(amidoamine)s: Past, present, and perspectives. J. Polym. Sci. Part A: Polym. Chem. 2013, 51, 2319-2353.

(40) Li, D.; Bu, Y.; Zhang, L.; Wang, X.; Yang, Y.; Zhuang, Y.; Yang, F.; Shen, H.; Wu, D. Facile Construction of $\mathrm{pH}$ - and RedoxResponsive Micelles from a Biodegradable $\operatorname{Poly}(\beta$-hydroxyl amine) for Drug Delivery. Biomacromolecules 2016, 17, 291-300.

(41) Yu, Q.; Zhang, Y.; Wang, H.; Brash, J.; Chen, H. Anti-fouling bioactive surfaces. Acta Biomater. 2011, 7, 1550-1557.

(42) Wible, B. C.; Gooden, C.; Saucier, N.; Borsa, J. J.; Cummings, L. S.; Cho, K. H. Ethylene-Vinyl Alcohol Copolymer Endobiliary Obliteration of Hepatic Segments in a Patient with Isolated Bile Leaks. J. Vasc. Interv. Radiol. 2014, 25, 1821-1825.

(43) Harter, J. G. The Status of Dimethyl Sulfoxide from the Perspective of the Food and Drug Administration. Ann. N.Y. Acad. Sci. 1983, 411, 1-5.

(44) Zeng, H.; Hwang, D. S.; Israelachvili, J. N.; Waite, J. H. Strong reversible $\mathrm{Fe} 3+-$-mediated bridging between dopa-containing protein films in water. Proc. Natl. Acad. Sci. U.S.A. 2010, 107, 12850-12853.

(45) Han, L.; Lu, X.; Liu, K.; Wang, K.; Fang, L.; Weng, L.-T.; Zhang, H.; Tang, Y.; Ren, F.; Zhao, C.; Sun, G.; Liang, R.; Li, Z. MusselInspired Adhesive and Tough Hydrogel Based on Nanoclay Confined Dopamine Polymerization. ACS Nano 2017, 11, 2561-2574.

(46) Slowing, I. I.; Vivero-Escoto, J. L.; Wu, C.-W.; Lin, V. S.-Y. Mesoporous silica nanoparticles as controlled release drug delivery and gene transfection carriers. Adv. Drug Deliv. Rev. 2008, 60, 1278-1288.

(47) Tang, F.; Li, L.; Chen, D. Mesoporous Silica Nanoparticles: Synthesis, Biocompatibility and Drug Delivery. Adv. Mater. 2012, 24, $1504-1534$

(48) Ranucci, E.; Spagnoli, G.; Ferruti, P.; Sgouras, D.; Duncan, R. Poly(amidoamine)s with potential as drug carriers: degradation and cellular toxicity. J. Biomat. Sci.Polym. E. 1991, 2, 303-315.

(49) Li, X.; Wang, Y.; Chen, J.; Wang, Y.; Ma, J.; Wu, G. Controlled Release of Protein from Biodegradable Multi-sensitive Injectable Poly(ether-urethane) Hydrogel. Acs Applied Materials \& Interfaces 2014, 6, 3640-3647.

(50) Derici, H.; Yaman, I.; Kara, C.; Kamer, E.; Diniz, G.; Ortac, R. Simvastatin Improves Incisional Wound Healing in a Rat Model: An Experimental Study. Wounds 2012, 24, 195-200. 
(51) Wong, V. W.; Rustad, K. C.; Akaishi, S.; Sorkin, M.; Glotzbach, J. P.; Januszyk, M.; Nelson, E. R.; Levi, K.; Paterno, J.; Vial, I. N.; Kuang, A. A.; Longaker, M. T.; Gurtner, G. C. Focal adhesion kinase links mechanical force to skin fibrosis via inflammatory signaling. Nat. Med. 2012, 18, 148-152.

(52) Olbrich, K. C.; Meade, R.; Bruno, W.; Heller, L.; Klitzman, B.; Levin, L. S. Halofuginone Inhibits Collagen Deposition in Fibrous Capsules Around Implants. Ann. Plas. Surg. 2005, 54, 293-296. 